\title{
Weight bearing through flexed upper limbs in quadriplegics with paralyzed triceps brachii muscles
}

\author{
LA Harvey*,1 and J Crosbie ${ }^{1}$ \\ ${ }^{1}$ School of Physiotherapy, University of Sydney, Australia
}

\begin{abstract}
Study design: A biomechanical analysis of lifting through flexed and extended elbows in C5 and C6 quadriplegics.

Objective: To determine the mechanisms used by $\mathrm{C} 5$ and $\mathrm{C} 6$ quadriplegics to prevent elbow collapse when bearing weight through flexed upper limbs.

Setting: A biomechanics laboratory.

Methods: Six motor complete C5 and C6 quadriplegic subjects with paralysis of their triceps brachii muscles were recruited. A three dimensional kinematic and kinetic analysis of the upper limbs was performed whilst subjects attempted to lift their body weight through their upper limbs under four different conditions. In one condition subjects lifted with their hands placed at the same height as the seat upon which they were sitting, whilst in the other three conditions subjects lifted with their hands placed on blocks of various heights. The four different conditions required subjects to bear weight through their upper limbs with their elbows initially flexed between 15 and $40^{\circ}$.

Main outcome measures: Angular displacements and corresponding moments about the shoulder, elbow and wrist joints. In addition, EMG data were collected from the upper pectoralis, anterior deltoid and biceps brachii muscles during all lifts and expressed as a percentage of maximal isometric voluntary contractions.

Results: As block height and initial elbow flexion increased, subjects lifted progressively less weight. However, even under the high block conditions when subjects' elbows were initially flexed up to $40^{\circ}$, subjects lifted a mean \pm SD of $43 \% \pm 20.4$ of their seated body weight with one subject lifting $76 \%$ of his seated body weight. Subjects lifted by generating shoulder and wrist flexor moments.

Conclusion: Quadriplegics with paralyzed triceps brachii muscles can bear moderate and sometimes substantial weight through flexed elbows. This is largely achieved by the generation of shoulder flexor moments.
\end{abstract}

Keywords: tetraplegia; transfer; upper limb function; rehabilitation

\section{Introduction}

C5 and C6 quadriplegics have paralysis of their trunk and lower limb muscles and consequently must rely on their upper limbs to prop, lift and move their bodies in order to transfer and perform an array of other gross motor tasks necessary for daily living. For this reason their ability to bear weight and/or lift their bodies using their upper limbs is fundamental to their ongoing independence and mobility. However, such a simple task as bearing weight through the upper limbs (ie, performing a weight relief maneuver) is difficult for these patients due to the paralysis of major upper limb muscles, particularly the triceps brachii muscle.

It is widely believed that, in the absence of innervated triceps brachii muscles, complete C5 and C6 quadriplegics can only support their body weight

*Correspondence: L Harvey, School of Physiotherapy, University of Sydney, P.O. Box 170, Lidcombe, NSW, 1825, Australia through their upper limbs by locking their elbows into a fully extended position. ${ }^{1-3}$ It has been assumed that full elbow extension prevents the elbows from collapsing by ensuring that the weight force vector passes posterior to the elbow, thus tending to extend it. ${ }^{4}$ However careful observation reveals that $\mathrm{C} 5$ and C6 quadriplegics often prop and support at least some of their body weight through flexed elbows. This is particularly evident when these patients attempt to lift or support their bodies with their hands placed higher than the seat upon which they are seated. Such a hand position requires these patients to commence and sometimes complete weight bearing with their elbows flexed. The ability of C5 and C6 quadriplegics to bear weight through flexed elbows has recently been verified kinematically, and EMG studies indicate that the upper pectoralis and the anterior deltoid muscles are active during equivalent weight bearing tasks. ${ }^{5-7}$ 
These muscles can extend the elbow in the absence of innervated triceps brachii muscles by appropriately rotating the proximal end of the upper arm in relation to the lower arm when the hand is fixed.

The precise mechanisms that enable C5 and C6 quadriplegics to bear weight through flexed elbows has not yet been elucidated. Therefore the aim of this study is to determine the mechanisms used by $\mathrm{C} 5$ and C6 quadriplegics to bear weight through their upper limbs from various initial positions of elbow flexion. In order to manipulate elbow angle, subjects were required to lift their weight with their hands placed on different sized blocks. The blocks necessitated that subjects commence lifting with the elbows in varying degrees of flexion. Consequently, shoulder position was also manipulated by the blocks.

\section{Methods}

Five motor complete $\mathrm{C} 6$ quadriplegics and one motor complete C5 quadriplegic (ie, with A and B impairment scores according to the American Spinal Injuries Association's standards for neurological classification) ${ }^{8}$ were selected for inclusion in this study. Subjects were male and of mean \pm SD age, weight and height of $34 \pm 5.3$ years, $74 \pm 17.7 \mathrm{~kg}$ and $177 \pm 8.2 \mathrm{~cm}$, respectively. Subjects had sustained their injuries $9 \pm 6.8$ years prior to testing. Subjects had either no muscle activity or only flickers of activity (grade 1/5) in their triceps brachii and wrist flexor muscles. The strength of subjects' shoulder adductor, flexor and extensor muscles varied between grade $2 / 5$ and $4 / 5$. All subjects had grade $4 / 5$ or $5 / 5$ in their elbow flexor muscles. The strength of subjects' wrist extensor muscles varied between $1 / 5$ and 5/5. All subjects had mild to moderate spasticity affecting the paralyzed muscles. Informed consent was obtained from each subject and the project was approved by the University of Sydney's Human Ethics Committee.

Subjects were required to sit on a wooden box $(500 \times 250 \mathrm{~mm})$ positioned on the floor and instrumented with four load cells (ADM Systems, Pty. Ltd., Seaford, Vic, Australia). The box was positioned between and level with two force platforms (OR6-51000, Advanced Mechanical Technology, Inc., Watertown, MA, USA) mounted on the floor. Subjects placed their hands on the force platforms to lift. The force platforms measured the $x, y$ and $z$ components of the forces and moments under each hand, whilst the seat measured the vertical ground reaction force under the buttocks. All force data (ie, from the instrumented seat and from the two force platforms) were amplified and collected by a personal computer using commercial software (BEDAS-2; Advanced Mechanical Technology Inc., Watertown, MA, USA) at a sampling frequency of $120 \mathrm{~Hz}$.

The testing protocol required subjects to lift six times under four different conditions, namely with their hands positioned directly on the two force platforms and on three pairs of blocks of varying heights positioned on the force platforms (low block condition $=45 \mathrm{~mm}$; medium block condition $=90 \mathrm{~mm}$; high block condition $=135 \mathrm{~mm}$ ). The order of testing was randomized. Exact hand position and speed of movement were not standardized, although subjects were instructed to lift as high as possible and hold the lift for approximately $2 \mathrm{~s}$. Prior to all testing subjects were given an opportunity to practise and familiarize themselves with the testing procedures. Eight seconds of data were collected for each lift and subjects were given approximately $5 \mathrm{~min}$ rest between each set of six lifts in order to minimize fatigue.

Twenty-four reflective markers were placed over the upper limbs, head and trunk of subjects. Marker triads were located in the middle third of the following segments: lower arm, upper arm, sacrum and head (total of 18 markers). Additional markers were placed over each scapula, the spinous process of the 7 th cervical vertebrae (CV7), the 4th and 12th thoracic vertebrae (TV4, TV12), and over the sternum. In order to prevent error due to skin movement relative to the underlying bone, markers were not placed directly over joints. ${ }^{9}$ Instead, the position of the shoulder, elbow and wrist joints were determined with the use of transform coordination matrices that defined the location of the joint centers in relation to adjoining marker triads.

Lifts were filmed at $60 \mathrm{~Hz}$ using six synchronized, COHU high performance video cameras (Motion Analysis Corporation, Santa Rosa, CA, USA). Amass software (Adtech, Adelphi, MD, USA) was then used to generate three dimensional spatial coordinates for the centroid of each marker. These data were subsequently used to derive three-dimensional angular displacements of the shoulder, elbow and wrist joints. Specifically, joint rotations were computed about the anatomical axes of flexion and extension at each of these joints, as well as adduction and abduction about the shoulder. Video data collected in a standardized resting posture at the commencement of each testing session were used to define the neutral positions of the shoulder, elbow and wrist according to the guidelines set out by the American Academy of Orthopedic Surgery. ${ }^{10}$ Zero degrees shoulder flexion and abduction indicated that the upper arm was parallel to the CV7-TV4 segment, and $0^{\circ}$ elbow flexion indicated that the elbow was fully extended. Zero degrees wrist extension indicated that the hand was parallel to the lower arm.

The kinematic and force data were later merged and standard inverse dynamics were used to derive the moments acting about the shoulder, elbow and wrist joints. Joint moments were expressed as internal muscle moments and were normalized to each subject's body weight. Kinematic and kinetic data were time-normalized across the upward phase of the lift. The beginning of the upward phase of the lift was identified by the first detectable decrease in the vertical force under the subject's buttocks, and the end was identified by the point at which this force was least. 
An additional $0.5 \mathrm{~s}$ of data both before and after the lift per se were also included for analysis.

For each variable, ensemble averages of each subject's three most successful trials were calculated from the normalized data linearly interpolated to every $1 \%$ interval. Subjects' averaged data were then pooled to obtain the group average and standard error (SE).

In addition, throughout testing surface EMG was collected from the upper pectoralis, anterior deltoid and biceps brachii muscles. EMG signals were collected at $2 \mathrm{kHz}$ and initially amplified by isolated preamplifiers (model NT464, Neomedix Systems, NSW, Australia) before further amplification (model NT810, Neomedix Systems, NSW, Australia). At this point all data were band-passed filtered between 50 and $500 \mathrm{~Hz}$ using a digital 8th order Butterworth filter. The signal was then sampled by a 16-bit analog to digital converter and collected onto a personal computer. Subsequently the EMG signal was full-wave rectified, smoothed (with a $0.1 \mathrm{~s}$ running average), sampled at $20 \mathrm{~Hz}$ and expressed as a percentage of the peak processed EMG signal obtained during maximal isometric contractions of the relevant muscles. The median (and semi-interquartile range) and peak EMG of the upward phase of the lift were then determined.

\section{Results}

The kinematics and kinetics of the upper limbs were largely symmetrical and therefore, for simplicity, only the results of the right upper limb will be presented. The higher blocks positioned subjects' elbows in more flexion and their shoulders in more extension and abduction at the commencement of the lift, though there was little difference in initial elbow angle between the no block and low block conditions and between medium and high block conditions (see Table 1). Subjects lifted a mean of $97 \%$ to $98 \%$ of their seated weight during the no and low block conditions. During lifts from the medium and high blocks subjects lifted a mean of $59 \%$ and $43 \%$ of their seated body weight (see Table 2 for details), respectively. One particularly heavy C6 subject (total body mass $=103 \mathrm{~kg}$ ) lifted between $70 \%$ and $88 \%$ of his seated body weight for all conditions and two other subjects lifted $46 \%$ and
$54 \%$ of their seated body weight under the high block conditions. The vertical forces under subjects' hands mirrored these results, though not perfectly, as subjects probably varied the amount of weight born through their heels during lifts. Median (semi-interquartile range) duration of the upward phase of the lift for the no, low, medium and high block conditions were $0.7 \mathrm{~s}(0.4-1.7), 1.4 \mathrm{~s}(0.8-2.3), 1.8 \mathrm{~s}(1.3-2.6)$ and $1.6 \mathrm{~s}(1.0-2.6)$, respectively.

Under all conditions, subjects tended to flex their shoulders and extend their elbows (see Table 1). However, whilst they attained near full elbow extension during lifts under the no and low block conditions, they were $22 \pm 10.1$ and $34 \pm 9.7^{\circ}$ short of full elbow extension during lifts under the medium and high block conditions, respectively. The wrist joint flexed between 12 and $16^{\circ}$ from an extended position during all lifting conditions, though the wrist was initially more extended during the higher block conditions (see Table 1).

Increases in vertical forces under the hands were associated with increases in shoulder, elbow and wrist flexor moments (see Figure 1), in all four lifting conditions. Larger shoulder flexor moments were required to generate the same vertical forces under the hands for the higher block conditions. Nearly identical wrist and elbow flexor moments were generated during all lifting conditions after taking

Table 2 Subjects' seated mass (kg; determined at time of weighing) and the mean maximum mass lifted under the four different block conditions (expressed as a percentage of subjects' seat mass)

\begin{tabular}{lccccc}
\hline Subject & $\begin{array}{c}\text { Seated } \\
\text { mass } \\
(\mathrm{kg})\end{array}$ & $\begin{array}{c}\text { No } \\
\text { block } \\
(\%)\end{array}$ & $\begin{array}{c}\text { Low } \\
\text { block } \\
(\%)\end{array}$ & $\begin{array}{c}\text { Medium } \\
\text { block } \\
(\%)\end{array}$ & $\begin{array}{c}\text { High } \\
\text { block } \\
(\%)\end{array}$ \\
\hline 1 & 84 & 80 & 88 & 83 & 76 \\
2 & 68 & 99 & 99 & 88 & 54 \\
3 & 48 & 100 & 100 & 43 & 33 \\
4 & 50 & 100 & 94 & 42 & 29 \\
5 & 54 & 98 & 99 & 71 & 46 \\
6 & 50 & 98 & 98 & 24 & 19 \\
\hline
\end{tabular}

Table 1 Mean \pm SE initial and final shoulder flexion and abduction angles, elbow flexion angles and wrist extension angles for the four lifting conditions. See text for definitions of neutral positions

\begin{tabular}{|c|c|c|c|c|c|c|c|c|}
\hline & \multicolumn{2}{|c|}{ No blocks } & \multicolumn{2}{|c|}{ Low blocks } & \multicolumn{2}{|c|}{ Medium blocks } & \multicolumn{2}{|c|}{ High blocks } \\
\hline & Initial & Final & Initial & Final & Initial & Final & Initial & Final \\
\hline $\begin{array}{l}\text { Shoulder } \\
\text { flexion }\end{array}$ & $\begin{array}{l}5.0 \\
(5.49)\end{array}$ & $\begin{array}{l}12.7 \\
(2.18)\end{array}$ & $\begin{array}{l}-2.6 \\
(4.27)\end{array}$ & $\begin{array}{l}8.1 \\
(2.75)\end{array}$ & $\begin{aligned} &- 15.7 \\
&(5.24)\end{aligned}$ & $\begin{array}{r}-6.8 \\
(4.08)\end{array}$ & $\begin{array}{r}-18.8 \\
(6.46)\end{array}$ & $\begin{array}{r}-18.0 \\
(6.83)\end{array}$ \\
\hline $\begin{array}{l}\text { Shoulder } \\
\text { adduction }\end{array}$ & $\begin{aligned}-10.8 \\
(2.34)\end{aligned}$ & $\begin{array}{c}-15.1 \\
(2.85)\end{array}$ & $\begin{array}{l}-6.6 \\
(10.16)\end{array}$ & $\begin{array}{c}-13.7 \\
(9.47)\end{array}$ & $\begin{array}{l}-2.1 \\
(8.17)\end{array}$ & $\begin{array}{c}-8.8 \\
(7.24)\end{array}$ & $\begin{array}{l}12.7 \\
(7.23)\end{array}$ & $\begin{array}{l}7.4 \\
(7.15)\end{array}$ \\
\hline $\begin{array}{l}\text { Elbow } \\
\text { flexion }\end{array}$ & $\begin{array}{l}14.5 \\
(8.25)\end{array}$ & $\begin{array}{l}3.6 \\
(3.96)\end{array}$ & $\begin{array}{l}20.7 \\
(7.47)\end{array}$ & $\begin{array}{l}1.8 \\
(3.81)\end{array}$ & $\begin{array}{l}39.0 \\
(5.58)\end{array}$ & $\begin{array}{l}21.6 \\
(10.05)\end{array}$ & $\begin{array}{l}39.6 \\
(6.35)\end{array}$ & $\begin{array}{l}34.2 \\
(9.65)\end{array}$ \\
\hline $\begin{array}{l}\text { Wrist } \\
\quad \text { extension }\end{array}$ & $\begin{array}{l}75.4 \\
(3.88)\end{array}$ & $\begin{array}{l}63.5 \\
(5.18)\end{array}$ & $\begin{array}{l}73.7 \\
(9.03)\end{array}$ & $\begin{array}{l}61.8 \\
(5.36)\end{array}$ & $\begin{array}{l}79.0 \\
(5.69)\end{array}$ & $\begin{array}{l}63.6 \\
(4.95)\end{array}$ & $\begin{array}{l}82.0 \\
(3.93)\end{array}$ & $\begin{array}{l}70.0 \\
(5.20)\end{array}$ \\
\hline
\end{tabular}


into account the differences in the weight lifted between conditions. Shoulder adductor moments increased during lifts under the no and low block conditions. However, these moments were substantially less using the medium blocks and almost non existent during lifts from the high blocks (see Figure $1)$.

Lifting conditions did not significantly affect mean or peak EMG activity in the upper pectoralis, anterior deltoid and biceps brachii muscles $(P<0.05$; see Figures 1 and 2). Median mean EMG activity in the upper pectoralis and anterior deltoid muscles ranged between $41 \%$ and $64 \%$ MVC, and median peak EMG activity ranged between $76 \%$ and $99 \%$ MVC, respectively. Median mean EMG activity in the biceps brachii muscles was less than 2\% MVC in all lifting conditions. In all three muscle groups EMG peaked towards the end of the lift when shoulder flexor and adductor and elbow flexor moments were greatest. (a)

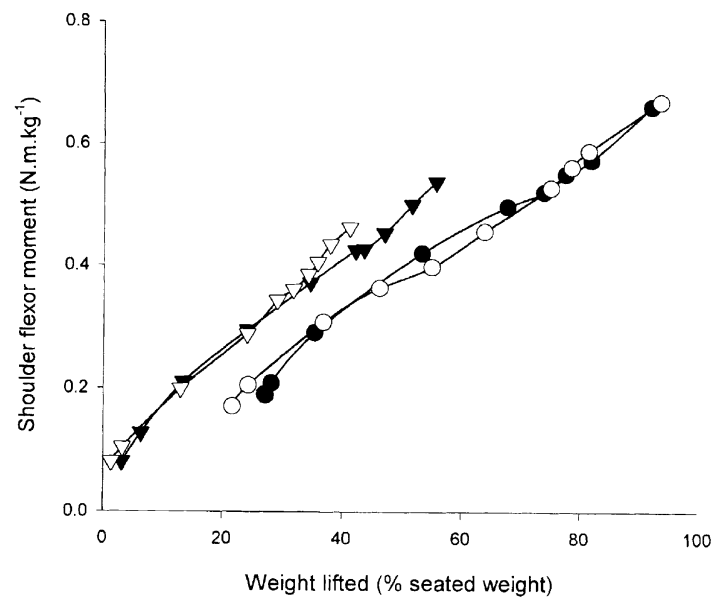

(c)

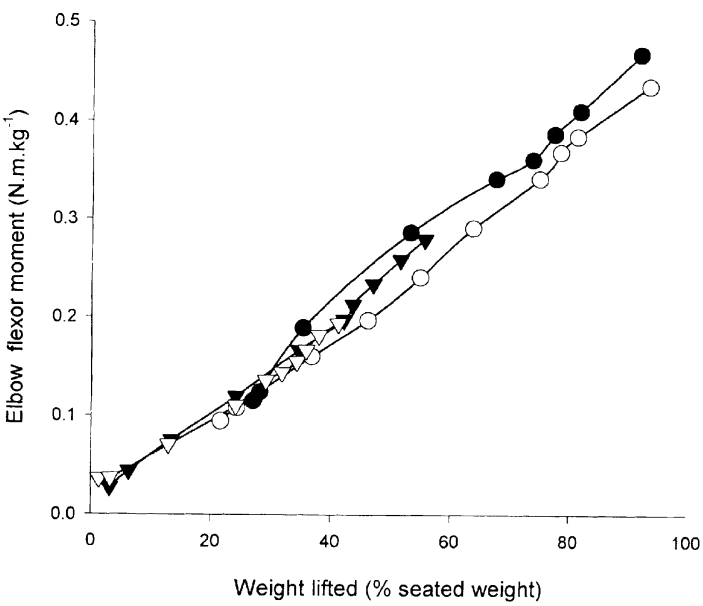

(b)

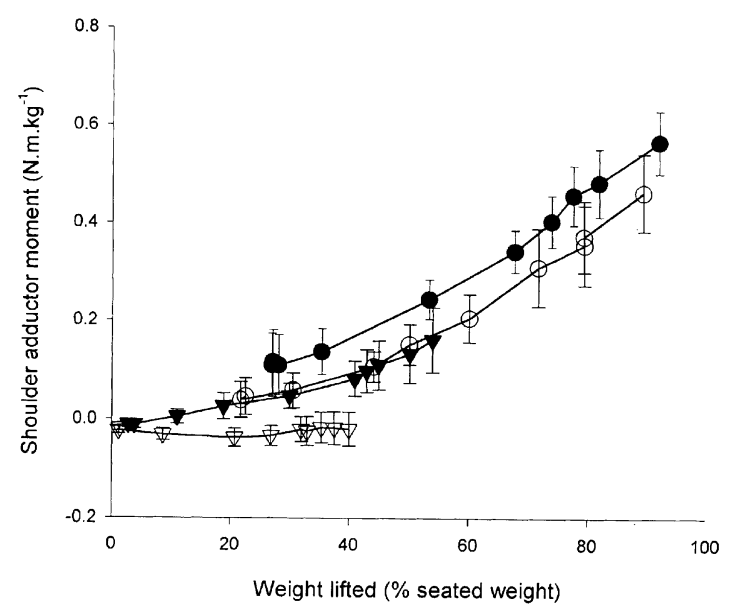

(d)

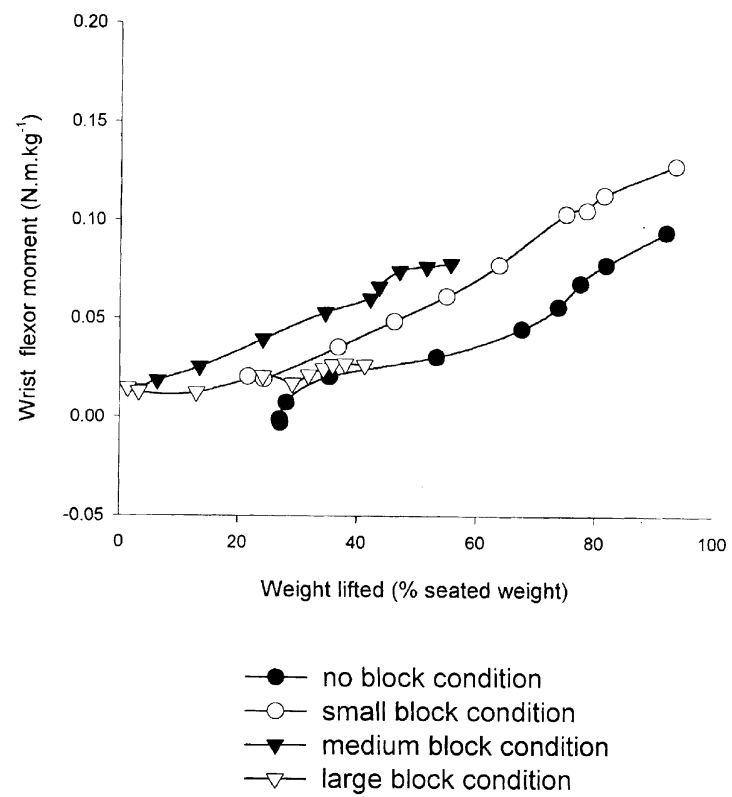

Figure 1 Mean \pm SE shoulder flexor moments (a), shoulder adductor moments (b), elbow flexor moments (c) and wrist flexor muscle moments (d) versus seated body weight for the four different lifting conditions. All data is normalized for lifting time and expressed as internal muscle moments in relation to body mass (N.m.kg ${ }^{-1}$ ). The error bars have been omitted from graphs (a), (c) and (d) for clarity 


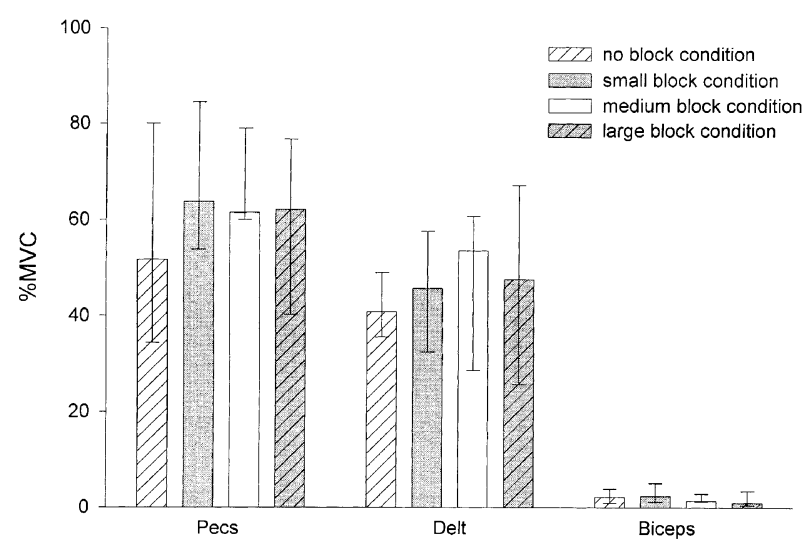

Figure 2 Median (and semi-interquartile range) mean EMG (expressed as \% MVC) for the sternal head of the pectoralis major (pecs), anterior deltoid (delt), and biceps brachii (biceps) muscles for lifts under the no block, low block, medium and high block conditions

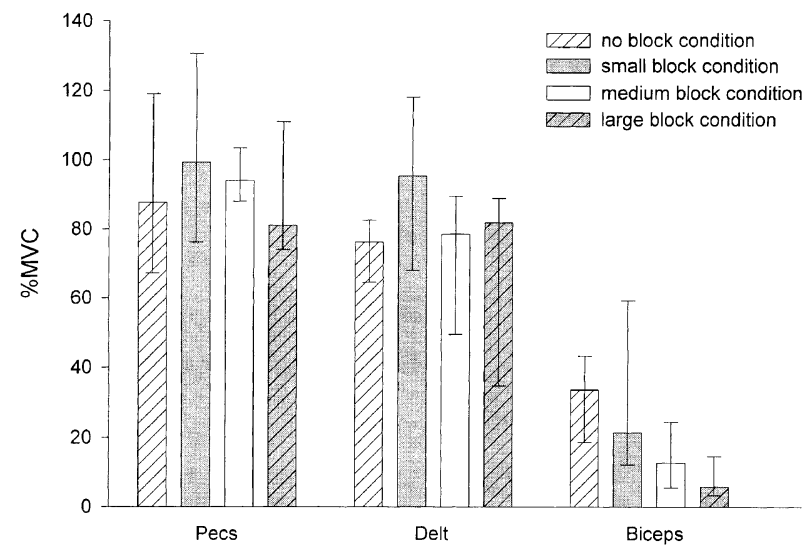

Figure 3 Median (and semi-interquartile range) peak EMG (expressed as \% MVC) for the sternal head of the pectoralis major (pecs), anterior deltoid (delt), and biceps brachii (biceps) muscles for lifts under the no block, low block, medium and high block conditions

\section{Discussion}

C5 and C6 quadriplegics have paralysis of their triceps brachii muscles. These muscles are generally considered to be primarily responsible for controlling and extending the elbow. ${ }^{11,12}$ However, this study shows that despite paralysis of the triceps brachii muscles, C5 and C6 quadriplegics are capable of stabilizing their elbows in a flexed position and bearing at least some and sometimes substantial weight through their upper limbs. This finding is contrary to the common belief that C5 and C6 quadriplegics are dependent on locking their elbows into a fully extended position in order to stabilise their elbows and prevent them from collapsing when bearing weight through the upper limbs..$^{1-3}$ This study is the first to provide an integrated kinematic, kinetic and EMG analysis of this skill.

The results indicate $\mathrm{C} 5$ and $\mathrm{C} 6$ quadriplegics bear weight through flexed elbows by generating quite large shoulder and to a lesser extent wrist flexor moments. Shoulder flexor and wrist flexor moments, can extend or stabilize the elbow by their rotatory action on the proximal end of the upper arm and distal end of the lower arm, respectively. An analogous situation has been observed in the lower limbs of patients unable to generate knee extensor moments. ${ }^{13}$ These patients use hip and ankle extensor moments to extend the knee whilst bearing weight through a fixed foot.

The EMG results indicate that the shoulder flexor moments were generated primarily by active contraction of the anterior deltoid muscle and/or the upper pectoralis muscles-both powerful shoulder flexor muscles. ${ }^{14}$ The importance of these two muscle groups for extending the elbow in quadriplegics with paralyzed triceps brachii muscles has been verified in other recent EMG studies. ${ }^{6,7}$ In contrast, wrist flexor moments must have originated from the passive stretch of soft tissue structures spanning the flexor aspect of the wrist, as all subjects had paralysis of wrist and finger flexor muscles and were therefore unable to actively contract these muscles in order to generate wrist flexor moments.

Even though subjects' elbows tended to extend during lifting, subjects generated elbow flexor moments under all lifting conditions. Any tendency for the elbow to flex due to elbow flexor moments must have been counteracted by the tendency for the elbow to extend under the influence of shoulder and wrist flexor moments and at times shoulder adductor moments. There was little mean EMG activity in the biceps brachii muscle. Possibly therefore, a large component of the elbow flexor moments originated from the stretch of soft tissue structures spanning the flexor aspect of the elbow, rather than active contraction of the biceps brachii muscle. The stretch in these structures would have increased as the elbow extended.

Subjects generated smaller shoulder adductor moments as block height and consequently initial elbow flexion increased. Possibly the position of the shoulder associated with the higher blocks placed the upper pectoralis muscles and other shoulder adductor muscles in a lengthened position and thereby made it biomechanically difficult for these muscles to generate shoulder adductor moments despite high levels of activation. However, it is unlikely that this factor alone accounted for such a dramatic decrease in the shoulder adductor moments with the higher blocks. A more likely explanation is that under the high block conditions shoulder adductor moments could not effectively reduce the load on the seat due to the extended position of the shoulder, and therefore the subject elected not to generate such moments. Instead the upper pectoralis muscles were recruited to flex the shoulder. 
It is therefore apparent from this study that C5 and C6 quadriplegics can stabilize a flexed elbow and bear weight through their upper limbs despite paralysis of their triceps brachii muscles. This is primarily achieved by the generation of shoulder flexor moments and to a lesser extent wrist flexor moments.

\section{Acknowledgements}

The Motor Accident Authority of NWS, Australia, (Shamay Ng, Darren Dawson).

\section{References}

1 Grover J, Gellman H, Waters RL. The effect of a flexion contracture of the elbow on the ability to transfer in patients who have quadriplegia at the sixth cervical level. J Bone Joint Surg (Am) 1996; 78: $1397-1400$.

2 Somers MF. Spinal cord injury: functional rehabilitation. Norwalk, Conn.: Appleton and Lange, 1992.

3 Bromley I. Tetraplegia and Paraplegia: a guide for physiotherapists. Edinburgh, New York: Churchill Livingstone, 1976.

4 Perry J. Normal upper extremity kinesiology. Phys Ther 1978; 58: $265-278$.
5 Allison GT, Singer KP, Marshall RN. Transfer movement strategies of individuals with spinal cord injuires. Disabil Rehabil 1996; 18: $35-41$

6 Gefen JY, Gelmann AS, Herbison GJ, Cohen ME, Schmidt R. Use of shoulder flexors to achieve isometric elbow extension in C6 tetraplegic patients during weight shift. Spinal Cord 1997; 35: $308-313$.

7 Marciello MA, Herbison GJ, Cohen ME, Schmidt R. Elbow extension using anterior deltoids and upper pectorals in spinal cord-injured subjects. Arch Phys Med Rehabil 1995; 76: 426-432.

8 Ditunno JF, Young W, Donovan WH, Creasey G. The international standards booklet for neurological and functional classification of spinal cord injury. Paraplegia 1994; 32: 70-80.

9 Cappozzo A et al. Position and orientation in space of bones during movement: Experimental artefacts. Clin Biomech 1996; 11: $90-100$.

10 American Academy of Orthopaedic Surgeons. Joint motion. Method of measuring and recording. Illinios: American Academy of Orthopaedic Surgeons, 1965.

11 An KN et al. Muscles across the elbow joint: a biomechanical analysis. J Biomechanics 1981; 14: 659-669.

12 Buchanan TS, Almdale DP, Lewis JL, Rymer WZ. Characteristics of synergic relations during isometric contractions of human elbow muscles. J Neurophys 1986; 56: $1225-1241$.

13 Winter RA. Overall principle of lower limb support during stance phase of gait. J Biomech 1980; 13: 923 - 927.

14 Kendall FP, Kendall McCreary E, Provance PG. Muscles. Testing and function. Baltimore: Williams and Wilkings, 1993. 